\title{
EL VÍNCULO SUSTANCIAL Y LAS MÓNADAS EN LEIBNIZ
}

Sergio Rodero

Universidad de Granada

Es en los últimos años del sistema leibniziano cuando se da forma y consideración al concepto físico -y fisiológico- de organismo. Este concepto orienta en cierto modo la interpretación de la doctrina de las mónadas, haciendo a los vivos, y en particular a los animales, el análogo por excelencia de las sustancias verdaderas. El concepto de "organismo" juega de facto un papel analítico en el fundamento de una representación "monadológica" del sistema de la naturaleza. Correlativamente, esta representación es sin duda apelada para especificar los elementos de base de la teoría del organismo, al mismo tiempo que ésta debe desplegarse en el plano de una explicación científica de los fenómenos. Es en este contexto que surge la teoría del vinculum substantiale, hipótesis sui generis elaborada por el Padre Bartholomeus des Bosses y se refiere a una conciliación posible del sistema de las mónadas y del dogma de la transustanciación. Si bien es cierto que el vínculo sustancial es una doctrina escolástica, retomada, eso sí, ahora, por Des Bosses y el propio Leibniz. ¿Podemos inferir de esa hipótesis de Des Bosses aparentemente ad hoc los elementos de un modelo subsidiario, susceptible de "sustancializar" el orden de los fenómenos orgánicos característicos de los autómatas vitales o vivos?

Pues si desarrollamos la versión monadológica del sistema leibniziano, los Principios de la naturaleza y de la gracia (obra de 1714) se basan sobre la distinción de las sustancias simples y compuestas para precisar los elementos constitutivos de una teoría de los vivos superiores. Las sustancias simples (las mónadas) dotadas de percepción y apetición, aparecen a la vez como las unidades verdaderas requeridas para rendir cuenta de los fenómenos, y como los principios que sostienen la actio vital. Es muy interesante que el desenvolvimiento analógico de la jerarquía de las

Philosophica, 37, Lisboa, 2011, pp. 73-83 
mónadas se funde sobre la serie viva -alma- espíritu, y que en virtud de esta doctrina de las sustancias simples, esté establecido que "toda la naturaleza está llena de vida”. ${ }^{1}$ Es en la relación de la mónada con el cuerpo donde se expresa más directamente que la percepción aparece como "representación del compuesto o de lo que está fuera en lo simple", y que las apeticiones hacen el papel de "principios del cambio"2. Las variaciones internas de las mónadas consisten, en efecto, en la variedad de las relaciones que las vinculan con los compuestos. Ellas simbolizan con los compuestos, lo que es un tipo de relación primordial muy interesante.

Ahora bien, consideremos más precisamente la relación de composición y de variación que hace el contenido interno representativo de la mónada. El corazón de la doctrina monadológica leibniziana tiende a la tesis de la correspondencia entre el contenido representativo y el cuerpo orgánico propio de cada mónada. O si no este cuerpo orgánico no es conocido como tal por la mediación analógica de los fenómenos de la vida animal. Pero Leibniz introduce la tesis significativa y aparentemente problemática según la cual cada mónada de un cierto nivel de perfección "es el centro de una sustancia compuesta (como, por ejemplo, de un animal) y el principio de su unicidad". ${ }^{3}$ Toda sustancia compuesta consiste en una masa extensiva sujeta a una forma de agregación. Se trata de la determinación de la mónada central del cuerpo propio lo que constituye el foco de unificación y de integración de la pluralidad de las vidas elementales correspondientes a la masa compuesta de una pluralidad de mónadas: las acciones propias correlativas de estas diversas mónadas en perpetuo cambio las unas en relación con las otras -las unas reaccionando sobre las otras y cada una constituyendo un "espejo viviente o dotado de acción interna"4 - determinan los estados del cuerpo orgánico, donde el principio de organización y vitalidad reside en la mónada central. En definitiva, reside aquí el fundamento de la tesis según la cual el cuerpo orgánico es un autómata o una máquina de la naturaleza donde las partes más pequeñas poseen la característica de ser ellas mismas los órganos dotados

${ }^{1}$ Ver Leibniz, Principios de la naturaleza y de la gracia fundados en razón, Aptdo. 1, Robinet I, p. 27 (ed. castellana de Ángel Luis González, OFC, 2, p. 344).

${ }^{2}$ Véase Leibniz, Principios de la naturaleza y de la gracia, Aptdo. 2, Ibid., p. 29 (ed. castellana citada, p. 344).

${ }^{3}$ V. Leibniz, Principios de la naturaleza y de la gracia, Aptdo. 3, Robinet I, p. 31 (ed. de Ángel Luis González, $O F C$, 2, p. 344).

${ }^{4}$ Leibniz, Principios de la naturaleza... Aptdo. 3, Ibid., p. 31 (OFC, 2, pp. 344-345); Cfr. Monadología, Aptdo. 56, GP VI, p. 616 (OFC, 2, p. 336): “Ahora bien, esta ligazón o acomodamiento de todas las cosas creadas a cada una y de cada una a todas las demás, hace que cada sustancia simple tenga relaciones que expresan a todas las demás, y que por consiguiente sea un espejo vivo y perpetuo del universo”; también en Teodicea, Aptdos. 130, 360. 
de funcionalidad propia. ${ }^{5}$ Recíprocamente, en la Monadología, el filósofo alemán hará valer que detrás de toda parte aparentemente inerte de materia se halla un mundo de vivientes, incluso de animales, formando los sistemas de cuerpos orgánicos más o menos desarrollados que implican las formas de vida sutiles que para nosotros resultan imperceptibles. ${ }^{6} \mathrm{El}$ sistema integrado que Leibniz nos oferta toma más bien entonces la forma de una integración jerárquica de vivientes o vivos más o menos elementales:

Cada cuerpo viviente posee una entelequia dominante, que es el alma en el animal; pero los miembros de este cuerpo vivo están llenos de otros seres vivientes, plantas, animales, y cada uno de ellos tiene a su vez su entelequia o su alma dominante. ${ }^{7}$

Leibniz toma, no obstante, la precaución de subrayar, inmediatamente después, que esta integración de vivientes (o vivos) es una integración de principios formales, ya que las partes materiales implicadas pueden no contener ninguna permanencia y estar sometidas a un flujo perpetuo, sin que soporten de ninguna manera la implicación de las formas de vida las unas con relación a las otras ${ }^{8}$.

No hay duda siguiendo el análisis de los Principios de la naturaleza y de la gracia que el pasaje más significativo para la noción de cuerpo orgánico está constituido por el artículo 4 donde Leibniz presenta la jerarquía de los cuerpos orgánicos en la noción de vivo animal y establece las características funcionales de la unidad sosteniendo la sustancia compuesta:

Cada mónada, con un cuerpo particular, forma una sustancia viva. Así, no sólo hay vida por doquier, unida a los miembros u órganos, sino que también hay una infinidad de grados en las mónadas, al dominar más o menos unas sobre las otras. Pero cuando la mónada tiene órganos tan ajustados que mediante ellos hay relieve y distinción en las impresiones que reciben y, por consiguiente, en las percepciones que la representan (como, por ejemplo, cuando, mediante la figura de los humores de los ojos, se concentran los rayos de la luz y actúan con más fuerza), puede

\footnotetext{
${ }^{5}$ Cfr. igualmente, Leibniz, Monadología, Aptdo. 64, GP VI, 618 (OFC, 2, p. 337) donde la relación de la pluralidad de las mónadas imbricadas al cuerpo propio regido por una mónada central es relacionada más abstractamente con un artificio mecánico divino. Los cuerpos vivos son también máquinas en sus mínimas partes ad infinitum. También en Teodicea, Aptdos. 134, 146, 194.

${ }^{6}$ Leibniz, Monadología, Aptdos. 66-68, GP VI, p. 618 (OFC, 2, p. 338).

${ }^{7}$ V. Leibniz, Monadología, Aptdo. 70, GP VI, p. 619 (OFC, 2, p. 338).

${ }^{8}$ Ver Leibniz, Monadología, Aptdo. 71, Ibid.: "Los cuerpos están en un flujo perpetuo, al igual que los ríos, y sus partes entran y salen de ahí continuamente”.
} 
llegarse hasta el sentimiento, es decir, hasta una percepción acompañada de memoria; o sea, una percepción de la que durante largo tiempo perdura un cierto eco para dejarse oír ocasionalmente; y a ese viviente se le llama animal, así como a su mónada se le llama $a l m a .^{9}$

Siguiendo el esquema analítico, Leibniz anexiona las búsquedas preformacionistas de los modernos en contra de toda teoría de la producción de formas orgánicas por epigénesis. Su tesis es, en este sentido, la de un advenimiento de las plantas y los animales a partir de "vivientes preexistentes", que son, de hecho, simples "semillas preformadas"10, sujetas a metamorfosis en el momento de la concepción. De modo correlativo, la muerte consiste sólo en el paso a una forma más sutil, que recuerda la forma embrionaria anterior a la metamorfosis. Leibniz sostiene, en última instancia, una mutación de especie de los cuerpos orgánicos concernidos, en el momento de la concepción como en el de la muerte. Esta mutación de especie se concibe, no obstante, necesariamente según la analogía de variaciones materiales vinculadas a la conservación de funciones vitales. Así lo expresa Leibniz en los Principios de la naturaleza:

Los animales cambian, toman y dejan sólo partes, lo cual ocurre poco a poco y por pequeñas partículas insensibles, pero continuamente, en la nutrición; y de un sola golpe, de manera notable, aunque raramente, en la concepción y en la muerte, que les hace adquirir o perder mucho de una vez. ${ }^{11}$

Entonces la distinción con relación al preformacionismo contemporáneo es todavía más notable si se tiene en cuenta el hecho de que los animales espermáticos nacen y mueren, a consecuencia de metamorfosis que implican microorganismos proporcionalmente más sutiles, y esto en una progresión analítica indefinida de requisitos orgánicos subyacentes a todo autómata de la naturaleza. Solamente un ínfimo número de elegidos

\footnotetext{
${ }^{9}$ Véase Leibniz, Principios de la naturaleza y de la gracia, Aptdo. 4, Robinet I, pp. 33-35 (OFC, 2, p. 345). El punto de vista expuesto en la Monadología, Aptdos. 62-63, GP VI, pp. 617-618 (OFC, 2, p. 337), es bastante más exotérico, ya que cada mónada representa todo el universo, pero más distintamente el cuerpo que le corresponde de manera más particular, y del que ella es la entelequia. Igualmente, encontramos allí las definiciones de viviente y de animal, mas el cuerpo propio es siempre orgánico por la razón general que la mónada representa el universo regulado según un orden perfecto. Ver también Teodicea, Aptdos. 43, 400.

${ }^{10}$ Ver Leibniz, Principios de la naturaleza...Aptdo. 6, Robinet I, p. 41 (OFC, 2, p. 346); Cfr. igualmente, Monadología, Aptdos. 72-74, GP VI, p. 619 (OFC, 2, pp. 338-339). Ver también Teodicea, Aptdos. 86, 89, 90, 124; asimismo, recomendamos la lectura del Prefacio de la ed. de Gerhardt, GP VI, pp. 40ss).

${ }^{11}$ V. Leibniz, Principios de la naturaleza y de la gracia, Aptdo. 6, Ibid.
} 
pasa a un teatro más grande ${ }^{12}$. En definitiva, una ley de organización recompone constantemente las partes corporales teniendo en cuenta la estructura orgánica que se trata de preservar, de desarrollar o de transformar radicalmente.

Podríamos alegar, ciertamente, que, en ciertos textos, Leibniz está mostrando su preocupación a las teorías de la preexistencia de los gérmenes que se desarrollan posteriormente a Malpighi, Swammerdam o Leeuwenhoek. Entre otros, el ejemplo por excelencia de tal caución parece figurar en la Monadología. El filósofo de Leipzig presenta ahí, no obstante, su tesis de la transformación de un cuerpo orgánico preexistente como fundado en los "principios deducidos a priori", con los cuales pretende que los "razonamientos hechos a posteriori y extraidos de experiencias se acuerden perfectamente". ${ }^{13}$ Resta saber que es lo que retiene precisamente a las teorías contemporáneas de la generación. Los datos de observación parecen justificar el esquema analógico de una semilla que posee por preformación los rudimentos de la planta o del animal, cuya organización se manifestaría por emergencia. Y esta emergencia correspondería a una "gran transformación", es decir, a una metamorfosis que haría venir un organismo en cierto modo de "otra especie"14. La analogía a este respecto proviene de las metamorfosis de insectos. Ciertamente, no habría allí mónada sin cuerpo orgánico; por consiguiente, la agregación o la disolución de las partes orgánicas no puede ser más que parcial. Entendemos por ello que de un estadio al otro de la vida orgánica no subsisten más que las mismas partes materiales, por lo menos los modos de combinación de esas partes que forman una serie continua en base a ciertas rupturas aparentes..$^{15}$ Existiría una ley de despliegue de los fenómenos de los estados orgánicos que expresaría la ley interna de la mónada dominante, pero desde el punto de vista homólogo de las combinaciones de partes materiales. Ambas leyes, una conforme a las exigencias de la finalidad en el despliegue de las percepciones/apeticiones, la otra respondiendo a las exigencias de la causalidad eficiente en los cambios mecánicos, se corresponden armónicamente.

12 En la presentación de los Principios de la filosofía o Monadología, Aptdo. 75, GP VI, p. 620 (OFC, 2, p. 339), esta hipótesis exotérica de animales espermáticos que sostienen los animales espermáticos de nivel superior no es evocada.

13 Cfr. Leibniz, Monadología, Aptdo. 76, Ibid. Ver además Teodicea, Aptdo. 90.

14 V. Leibniz, Monadología, Aptdo. 74, Ibid. En Teodicea, Aptdos. 86,89.

15 En la Monadología, Aptdo. 77, Ibid., el filósofo alemán traduce de hecho sumariamente este requisito de un sujeto permanente de las mutaciones orgánicas aparentes: "Así puede decirse que no sólo el alma (espejo de un universo indestructible) es indestructible, sino también el animal mismo, aunque su máquina a menudo perezca en parte y pierda o adquiera despojos orgánicos”. 
En la famosa Correspondencia con Des Bosses se precisa la versión monadológica de la teoría del organismo, haciendo sitio al modelo controvertido del vinculum substantiale ${ }^{16}$. Ambos aspectos de esta contribución analítica merecen ser tratados ampliamente. Leibniz escribió a Des Bosses:

Cuando digo que no existe ninguna parte de la materia que no contenga mónadas, lo ilustro con el ejemplo del cuerpo humano o de otro animal, cualquier parte suya, sólida o fluida, contiene también en sí otros animales y vegetales (...). Así, la materia cambia como un río, permaneciendo la Entelequia mientras subsiste la máquina. La máquina tiene una Entelequia adecuada a sí y esta máquina contiene otras máquinas inadecuadas, cierto, a la Entelequia primera, pero dotadas de sus propias entelequias adecuadas a sí y separables de la primera. ${ }^{17}$

Leibniz sostiene que el animal está compuesto de una mónada dominante y de un cuerpo orgánico fenomenal, y que el organismo así constituido comprende, como partes integrantes, las máquinas orgánicas, ellas mismas compuestas de mónadas y de cuerpos propios, y susceptibles de descomposición ad infinitum. El caso del organismo es el de una pluralidad de mónadas en orden de subordinación y de integración parcial. Se reflejan en una máquina orgánica cuyo funcionamiento implica a las máquinas orgánicas subalternas. Otro modo de representar esto es considerar el cuerpo orgánico como "el resultado de innumerables sustancias completas, de las que cada una tiene su entelequia y su materia primera, pero ninguna de ellas está fijada perpetuamente a la nuestra". ${ }^{18} \mathrm{~A}$ este respecto, ninguna de estas mónadas subalternas juntadas a sus cuerpos orgánicos contribuye,

16 Sobre el vinculum substantiale en Leibniz véase: Maria Ramon Cubells, "Mónadas y compuestos”, en Éndoxa: Series Filosóficas, 4, 1994, UNED, Madrid, pp. 129-136; Brandon Look, Leibniz and the "vinculum substantiale", en Studia Leibnitiana, 30, Sonderheft, 1999; Brandon Look, Leibniz and the Substance of the Vinculum substantiale, en Journal of the History of Philosophy, 38, $\mathrm{n}^{\circ} 2,2000$, pp. 203-220; Alfonso Pérez de Laborda, Tiempo e Historia: Una filosofía del cuerpo, Ediciones Encuentro, Madrid, 2002 (cap. 3 especialmente); Brandon Look \& Donald Rutherford, The Leibniz-Des Bosses Correspondence, Yale UP., Yale, 2007; Juan Antonio Nicolás \& Maria Ramon Cubells, G.W. Leibniz: Obras filosóficas y cientificas, Comares, Granada, 2007 (2 ${ }^{\mathrm{a}}$ parte, pp. 153-467); Daniel Garber, Leibniz: Body, Substance, Monad, Oxford UP., Oxford, 2009.

17 Leibniz, Carta a B. Des Bosses, de 7 de marzo de 1706, GP II, pp. 305-306: empleamos aquí la edición de Juan A. Nicolás \& María Ramon Cubells, G.W. Leibniz. Obras filosóficas y científicas, Correspondencia I, vol. 14, Comares, Granada, 2007, p. 173.

18 Leibniz, Carta a Des Bosses, de 16 de octubre de 1706, GP II, p. 324 (Cfr. Juan A. Nicolás \& María Ramon Cubells, Ibid., p. 200). 
para una parte "esencial”, a la composición misma del organismo. Esto es conforme al modelo de la gota de aceite que Leibniz evoca con ocasión de rendir cuenta a la vez de la persistencia del animal a través de sus metamorfosis y de la relatividad de la relación a las máquinas subalternas. ${ }^{19}$ Imaginemos que un animal -dice Leibniz- viene a ser como una gota de aceite y el alma como un punto en la gota. Si se divide la gota en partes, siendo que cualquier parte vuelva a ser una gota con forma de globo, aquel punto existirá en alguna de las nuevas gotas. El punto-alma persistirá solamente en una de esas gotas. La dislocación de la máquina global no hace desaparecer ni el alma ni el organismo que ella gobierna: la máquina se repliega entonces sobre un organicidad elemental, y pierde de este modo casi totalmente la combinación de órganos que la definía, siendo que la ley de combinación asegura la continuidad entre el menor estado precedente a la concepción y el estado superior del organismo actualizado, pues entre éste y el estado menor resulta la muerte orgánica.

Leibniz introduce, pues, una alternativa que consiste en tratar el cuerpo como sustancia compuesta, siguiendo el sistema monadológico, como un puro conjunto de fenómenos, o, según el modelo del vínculo, como "una realidad de unión, que añade algo de absoluto (y por tanto sustancial), aunque sea efímero, a los elementos que se han de unir"20. El filósofo de Hannover añade que la transustanciación se situaría en el cambio de este absoluto por voluntad divina, al mismo tiempo que las afecciones monádicas que sirven de requisitos formales a la sustancia compuesta darían lugar a apariencias fenomenales congruentes. Así se concebiría la transformación de los fenómenos bien fundados en realidades sui generis, capaces de integrar los flujos de partes materiales que compondrían los agregados corporales e imponer una estructura formal de unificación de las mónadas subyacentes a la mónada dominante. Así lo expresa Leibniz en una misiva a Des Bosses:

Si faltase aquel vínculo sustancial de las mónadas, todos los cuerpos con todas sus cualidades no serían otra cosa que fenómenos bien fundados, como el arco Iris o la imagen en un espejo, en una palabra, serían sueños continuados perfectamente congruentes consigo mismos; y en esta unidad consistiría la realidad de estos fenómenos (...). Así pues, si el cuerpo es una sustancia, es una realización de los fenómenos que va más allá de la congruencia fenoménica ${ }^{21}$.

19 Véase Leibniz, Carta a Des Bosses, de 11 de marzo, de 1706, GP II, p. 306 (Cf. Juan A. Nicolás \& María Ramon Cubells, Ibid., p. 174).

${ }^{20}$ Véase Leibniz, Carta a Des Bosses, de 5 de febrero de 1712, GP II, p. 435 (Cf. Juan A. Nicolás \& María Ramon Cubells, Ibid., p. 358).

${ }^{21}$ V. Leibniz, Carta de Leibniz a Des Bosses, de 5 de febrero de 1712, GP II, pp. 435-436 (ed. española de Juan A. Nicolás \& María Ramon Cubells, Ibid., pp. 358-359). 
Entonces, el punto de vista fenomenista nos permite identificar las máquinas de la naturaleza, sus estructuras y sus procesos siguiendo las secuencias de estados materiales cuya ley de congruencia depende formalmente de la inherencia de percepciones/apeticiones en los sujetos monádicos. En cuanto al concepto de vínculo sustancial aplicado a la sustancia compuesta, nos permite representar el organismo como un agente autónomo susceptible de conservarse por un tiempo e imponiendo un orden causal único a las estructuras y procesos físicos que lo constituyen. Ésta es la tesis de B. Look. Ese agente poseería, en su realidad formal emergente, el poder de organizar sintéticamente a los vivos más elementales que constituirían los ingredientes dinámicos.

Look revela y resalta la novedad del vinculum substantiale, y es que el vínculo se refiere a la sustancia compuesta. ${ }^{22}$ El vínculo -para Lookestablecería la unicidad del agregado monádico y no respondería a la clásica cuestión de la unión alma-cuerpo en Leibniz. ${ }^{23}$ En este sentido estoy de acuerdo con la posición de Adelino Cardoso para contrariar a Look entendiendo que el vínculo sustancial es la respuesta sistemáticamente coherente del leibnizianismo a las cuestiones cruciales que la naturaleza coloca al hombre. La doctrina del vínculo sustancial da coherencia y sistematicidad a la filosofía monadológica leibniziana, que transporta consigo un proyecto de racionalidad con una ordenación orgánica de los saberes. ${ }^{24}$ Hay otra interpretación interesante, la reflejada por Robert Adams, que hace valer que Leibniz asimila de dos modos distintos los elementos doctrinales relativos a la restauración de las formas sustanciales aristotélico-escolásticas. ${ }^{25}$ Una de las vías de asimilación es “autónoma” y comporta las tesis remodeladas conforme a los conceptos monadológicos; la otra vía es "heterónoma" y contiene las conciliaciones y arreglos a favor de tesis que tratan de acercar el nuevo sistema de la naturaleza. ¿ ¿No sería éste el caso del modelo subsidiario representado por la noción de vínculo sustancial?

22 B. Look dirá: “...the vinculum substantiale is designed to resolve concern the unity and reality of composite substance”, en Brandon Look, op. cit., p. 157.

${ }^{23}$ Leibniz usa muy claramente la frase "unión metafísica sólo cuando habla de la relación entre mente y cuerpo"; y usa claramente la frase "unión real sólo cuando habla de las relaciones entre mónadas en una sustancia compuesta. Y textualmente dice Look a propósito de esto: the difference corresponds to the different metaphysical explanations in terms of mind and body, on the one hand, and in terms of monads, of he other hand”, en B. Look, op. cit., p. 93.

24 Adelino Cardoso, $O$ trabalho da mediaçäo no pensamento leibniziano, Ed. Colibrí, Lisboa, 2005, pp. 203-205.

25 V. R.M. Adams, Leibniz Determinist, Theist, Idealist, New York/Oxford University Press, New York, 1994, en particular, pp. 299-307. 
Ciertamente, como ya hemos dicho, en la Correspondencia con B. Des Bosses el filósofo germano específicamente piensa establecer una conciliación entre su filosofía y el dogma de la transustanciación. Desde este punto de vista, el modelo es concebido para preservar lo esencial de la doctrina monadológica. Es notable por otra parte que Leibniz hace depender este nuevo orden emergente de la voluntad divina allende las determinaciones del entendimiento supremo, el cual se presenta a los sujetos reales que son las mónadas y a las relaciones que emanan de él26. Todo pasa como si la intención transcendental sostenga e inscriba un orden orgánico "sustancial” en el curso mismo de los fenómenos. En estas condiciones, Leibniz argumenta como si presumiera que el orden empírico directamente pueda expresar el reino de los fines en la formación, la conservación y la disolución de los cuerpos.

A pesar de todo, es difícil no conceder al modelo subsidiario una cierta función epistemológica en la representación de los cuerpos compuestos como organismos. Así lo especifica Leibniz en Carta a Des Bosses:

Pienso que las Mónadas tienen siempre una existencia plena y no se pueden concebir como las partes que se dice que están en el todo en potencia. No veo tampoco que la Mónada dominante les quite existencia a las otras mónadas, ya que en realidad entre ellas no hay ningún comercio, sino tan sólo consenso (...) La dominación y la subordinación de las mónadas, considerada en las mismas mónadas, no consiste más que en los grados de perfección. ${ }^{27}$

En definitiva, la sustancia compuesta aparece como una sustancia que existiría por sí. Tiene mucho interés concebir que la relación de fundamento que iría de las mónadas a los fenómenos orgánicos podría así invertirse, por un tipo de representación metafórica, para arrastrar un análisis de las condiciones formales y materiales que dirigirían la existencia de lo vivo orgánico al plano mismo de los fenómenos. Esta representación reposaría sobre el concepto de forma fenomenal emergente, para

${ }^{26}$ Ver Anexo a la Carta de Leibniz a Des Bosses, de 5 de febrero de 1712, GP II, p. 438 (Cfr. ed. española de Juan A. Nicolás \& María Ramon Cubells, Ibid., p. 362): "Pero además de estas relaciones reales se puede concebir una más perfecta por la que de una multiplicidad de sustancias sale otra nueva. Y ésta no será un simple resultado, es decir, no constará solamente de las relaciones verdaderas o reales, sino que además le añadirá alguna nueva sustancialidad o vínculo sustancial, y no será efecto sólo del entendimiento divino, sino también de la voluntad divina”.

${ }^{27}$ Carta de Leibniz a Des Bosses, 16 de junio de 1712, GP II, p. 451 (ed. castellana de Juan A. Nicolás \& María Ramon Cubells, Ibid., p. 378). 
derivar en cierto modo en los requisitos monádicos variables según las conexiones espaciales y las sucesiones temporales orgánicamente vividas.

Esto nos lleva a la metáfora del eco que Leibniz utiliza en las últimas páginas de la Correspondencia con Des Bosses. Este modo de presentar las cosas permite concebir que la relación constitutiva del eco es natural y habitualmente conforme a las implicaciones de las leyes monádicas subyacentes, al mismo tiempo que parece expresarse de modo autónomo en el plano de los fenómenos. Mas lo importante es constatar que el eco posee un orden interno sui generis que puede servir para determinar los modos que le afectan en tanto que fenómeno bien fundado. El análisis así producido puede adecuadamente despuntar en dirección a las razones formales correspondientes al plano de las mónadas como los centros de fuerza. Se trataría de un uso analógico del vínculo sustancial "como principio de acción del compuesto" 28 , actualizando el orden específico de las manifestaciones físicas del organismo, lo que puede ser el sistema de requisitos formales acondicionado metafísicamente.

A modo de conclusión, diremos que en la época de Leibniz el iatromecanismo principalmente reinaba: era entonces la anatomía sutil que determinaba la concepción del ser vivo. El postulado de base era que el entendimiento humano, tomando apoyo sobre la observación microscópica y proyectando los modelos mecanicistas para rendir cuenta de las microestructuras, podría penetrar en las causas reales de los fenómenos. Si Leibniz dibuja para la medicina racional una metodología con propensión empirista en corrección de un modelo iatromecanista estricto, es sin duda que, según él, el análisis de los fenómenos vitales particulares exige, a consecuencia de su complejidad y a consecuencia de su diversidad, una lectura atenta y un desciframiento analítico de las apariencias sensibles a varios niveles: una explicación necesariamente provisional puede entonces apoyarse sobre la definición de propiedades funcionales específicas. Para que los fundamentos de la teoría fisiológica sean tomados en consideración en el sistema leibniziano de la naturaleza, los elementos de la medicina racional deben establecer los principios de una historia natural razonada de los fenómenos vitales, que escapará del esquematismo estructural estrecho, por así decir, de los modelos mecánicos. El modelo mecánico revisado de Leibniz, en la medida en que marcaba una orientación característica de búsqueda, se revelaba apto para desempeñar un papel crítico y para influir en el desarrollo ulterior de las teorías fisiológicas.

${ }^{28}$ Cfr. Leibniz, Carta de Leibniz a Des Bosses, de 13 de enero de 1716, GP II, p. 511 (Cfr. ed. castellana de Juan A. Nicolás \& María Ramon Cubells, Ibid., p. 454). 


\title{
RESUMEN
}

El vínculo sustancial es para Leibniz la relación en donde las sustancias simples (mónadas) se unen para integrarse en un compuesto del que surge una sustancialidad colectiva, y no es la mera adición de sustancialidades individuales. El vínculo es fundamental, determinante para el compuesto, pero no para lo simple. Lo conectivo se vuelve definitorio en lo compuesto, de manera que la ontología leibniziana apuesta fuertemente por lo simple, por lo individual, como actualización de lo infinitesimal.

Palabras-clave: Leibniz, vínculo sustancial, mónada, ontología, infinitesimal.

\begin{abstract}
The substantial vinculum is for Leibniz the relation where the simple substances (monads) join to create a compound from which a collective substantiality arises, and it is not the simple addition of individuals substantialities. The vinculum is essential, determinant for the compound, but not for the simple thing. The connective thing turns definitive in the compound thing, so that the ontology of Leibniz bets strongly for the simple thing, for the individual thing, as update of the infinitesimal thing.
\end{abstract}

Key-words: Leibniz, substantial vinculum, monad, ontology, infinitesimal thing. 